\title{
Deep views into living mice
}

Tool development facilitates deep-tissue photoacoustic imaging.

Deep-tissue imaging can yield crucial insights into biological processes as they occur in living organisms. For this reason, new tools for such intravital imaging are an area of intense research. However, most tissues are highly scattering to light, which limits the imaging depths or spatial resolution that can be achieved by most light-based optical approaches, including fluorescence and bioluminescence.

Paul Beard at University College London, Martin Pule of the Cancer Institute at University College London and their colleagues sought a different strategy to enable deep-tissue imaging of target cells. Beard recalls that the team wanted to develop genetic reporters for photoacoustic imaging (PAI), which is a modality that can potentially image 3-4 centimeters deep with high resolution. He notes that "if this could be achieved, it would offer a wealth of new opportunities to visualize cellular and genetic processes in vivo at the tissue, organ or even whole-body level in small mammals that would otherwise be impossible."

In PAI, pulsed-laser excitation is used to illuminate samples. Absorption of the light by chromophores within the tissue leads to the generation of ultrasonic waves, which are then detected and used to generate an image of the sample. PAI is often used to image an organism's vasculature in three dimensions, as endogenous hemoglobin provides good imaging contrast. However, genetically encoded probes for labeling specific cells in PAI have lagged behind analogous probes for fluorescence imaging.

One available probe is tyrosinase (Tyr), an enzyme that generates a pigment called eumelanin, which provides good contrast in PAI. According to Beard, Tyr is well suited for deep-tissue imaging because it is a mammalian protein that is likely to be well tolerated in cells and because it amplifies contrast by generating multiple eumelanin molecules per Tyr molecule. These eumelanin molecules then absorb strongly at the nearinfrared wavelengths used in deep-tissue imaging.

\section{GENOMICS}

\section{SPLITTING Cas 9}

Two research groups show that the CRISPR system can be regulated by

heterodimerizing a split Cas9.

Few other technologies have seen the rapid rise in uptake by the scientific community that the clustered, regularly interspaced, short palindromic repeats (CRISPR) system has enjoyed over the last 2 years.

First discovered in 1987 by a Japanese research group, these virus-derived repeat sequences separated by spacers in bacterial genomes received the name CRISPR in 2002 from researchers at Utrecht University in the Netherlands. For the next decade, the mechanism-which allowed guide RNAs (gRNAs) encoded in the CRISPR locus to target CRISPR-associated (Cas) nucleases to matching viral DNA sequences-was studied mainly by scientists interested in bacterial immunity. In 2012, work by Jennifer Doudna at the University of California, Berkeley, and Emmanuelle Charpentier, then at Umeå University in Sweden, showed that the system could be programmed so that the gRNA targets Cas 9 to any DNA sequence of interest.

Several papers in short succession at the beginning of 2013, including independent work from Doudna and Feng Zhang at the Broad Institute of MIT and Harvard, demonstrated that the CRISPR-Cas9 system can be used to edit mammalian genomes. And so the floodgates were opened. CRISPR-Cas9 has since been used to edit the genomes of many species, it has activated and repressed genes, and its mechanism has been scrutinized in detail.

Doudna and her team are still at the forefront of CRISPR-Cas9 research. One of their goals is to turn Cas9 'on' and 'off' in a particular type of tissue or cell or in response to an environmental condition. Looking at the three-dimensional structure of Cas9, her team noticed two separate structural regions. They reasoned that a Cas 9 split into those parts could be heterodimerized upon addition of the gRNA, which is known to bind both regions. 
Tyr had been used to generate PAI contrast in previous studies; however, the researchers developed the technology in order to label cells and follow them over several weeks in a living mouse. "The challenge [was] to modify the cell genome so as to achieve high eumelanin production for a detectable PA signal without disrupting the underlying cell biology and introducing toxicity," says Beard.

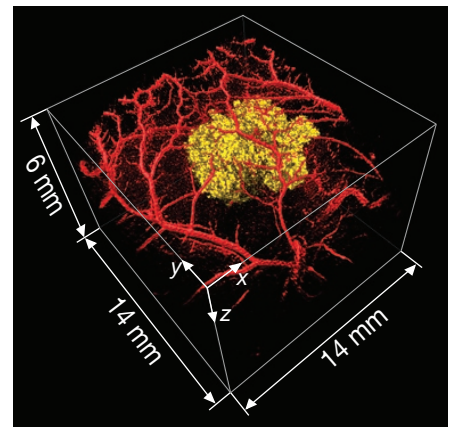

Volume-rendered image of Tyr-labeled cancer cells (yellow) after injection into a mouse. Vasculature is shown in red. Reproduced from Jathoul et al., Nature Publishing Group.
"This was addressed by transfecting the cells using a retroviral vector and carefully tuning the expression levels to strike the optimal compromise between signal and metabolic burden."

In addition to optimizing Tyr labeling, the team also developed a highly sensitive photoacoustic scanner for imaging mice. According to Beard, this scanner uses an entirely new type of detector that is orders of magnitude more sensitive than conventional detectors. In addition, the team used a novel signal processing method to compensate for distortions in the photoacoustic waves as they travel to the detector.

The combination of the new scanner and optimized labeling yielded striking results in terms of imaging depth and resolution. Using their approach, the researchers were able to inoculate Tyrlabeled cancer cells into mice and image the cells over several weeks with image clarity that amazed even the researchers themselves. Indeed, Beard was "surprised at how well the Tyr-labeled cells could also be visualized against the large background signal provided by hemoglobin, even at depths approaching 1 centimeter." He notes that in the future, this imaging approach could help facilitate studies of cell growth, angiogenesis and cancer biology in vivo.

\section{Rita Strack}

\section{RESEARCH PAPERS}

Jathoul, A.P. et al. Deep in vivo photoacoustic imaging of mammalian tissues using a tyrosinase-based genetic reporter. Nat. Photonics 9, 239-246 (2015).

Graduate students Addison Wright and Samuel Sternberg designed and optimized a split-not a strict cleavage into two parts; they left intact the helical lobe that lies roughly in the middle of the peptide sequence-and then fused the very $\mathrm{N}$-terminal domain to the C-terminal nuclease region. They showed that, upon addition of gRNA, the two Cas9 parts assemble into an intact complex with cleavage activity similar to that of the wild-type protein. They also demonstrated that a truncated gRNA is no longer able to assemble the split Cas 9 but can still bring intact Cas 9 to its target sequence. This opens the door for future work to use peptide dimerization domains, rather than gRNA, to bring the Cas9 parts together in response to small molecules or light.

In recent work, Feng Zhang showed just such a peptide-driven dimerization. His team used a split separating the $\mathrm{C}$ - and $\mathrm{N}$-terminal Cas 9 domains and fused them with proteins that dimerize upon the addition of rapamycin. They eliminated background cleavage in the absence of rapamycin by shuttling the N-terminal part of Cas9 out of the nucleus with a nuclear export signal and ensuring that only the assembled complex would be transported back into the nucleus by outfitting the C-terminal fragments with two import signals. His team used this system for genome editing and inducible transcriptional activation.

Combining the truncated gRNA of Doudna's group with the localization-specific assembly of Zhang's team may make for a system with even higher specificity. Doudna sees potential therapeutic applications down the line. "What we would like to do with this activatable system is to use it to correct the mutations in relevant tissue types or alter gene expression in ways that would affect a disease state," she says.

Nicole Rusk

\section{RESEARCH PAPERS}

Wright, A.V. et al. Rational design of a split-Cas9 enzyme complex. Proc. Natl. Acad. Sci. USA 112, 29842989 (2015).

Zetsche, B. et al. A split-Cas9 architecture for inducible genome editing and transcription modulation. Nat. Biotechnol. 33, 139-142 (2015). 